\title{
In Vitro Characterization of the Molecular Machinery Regulating Umbilical Cord Blood Mesenchymal Stem Cell Angiogenesis: A Step Towards Multipotent Stem Cell Therapy for Vascular Regeneration
}

Santiago Roura ${ }^{1 *}$, Juli R Bagó2,3, Carolina Gálvez-Montón ${ }^{1}$, Jerónimo Blanco ${ }^{2,3}$ and Antoni Bayes-Genis ${ }^{1,4,5, *}$

${ }^{1}$ Heart Failure and Cardiac Regeneration (ICREC) Research Program, Health Research Institute Germans Trias i Pujol (IGTP), Badalona, Spain

${ }^{2}$ Cell therapy group, Institute for Advanced Chemistry of Catalonia, Barcelona, Spain

${ }^{3}$ Networking Biomedical Research Center on Bioengineering, Biomaterials and Nanomedicine (CIBER-BBN), Spain

${ }^{4}$ Cardiology Service, University Hospital Germans Trias i Pujol (HuGTiP), Badalona, Spain

${ }^{5}$ Department of Medicine, Universitat Autònoma de Barcelona, Barcelona, Spain

\begin{abstract}
Umbilical cord blood (UCB)-derived mesenchymal stem cells (MSCs) promote vascular growth in vivo. Here we examined the intracellular regulatory machinery involved in the in vitro angiogenic behaviour exhibited by UCBMSCs. Angiogenic activity was measuring in the standard Matrigel-based culture assay after treatment of cells with known modulators of early growth response factor (Egr-3) and endothelial cell (EC) angiogenesis. Egr-3 expression was assessed by quantitative RT-PCR and indirect immunofluorescence, and specifically abrogated using small interfering RNA (siRNA) technology. While addition of phorbol-12-myristate-13-acetate (PMA) promoted angiogenic capacity $(P<0.001)$, selective inhibitors of PKC/MAPK/ERK abrogated this capacity $(P=0.016)$ in UCBMSCs. Treatment with PMA increased Egr-3 mRNA and protein levels $(P<0.001)$. However, cyclosporine $\mathrm{A}(\mathrm{CsA})$ and vascular endothelial growth factor (VEGF) affected neither Egr-3 levels nor the formation of polygonal cell networks. PMA also induced ERK $1 / 2$ phosphorylation, which was abolished by the selective inhibitor U0126 ( $P=0.021$ and $P=0.014$, respectively). Marked inhibition of network-forming capacity was observed in siEgr-3-transduced cells $(P<0.001)$. Taken together, our results highlight that Egr-3 is commonly involved in the molecular machinery regulating mature $E C$ and multipotent MSC angiogenesis. This knowledge may be applied to increase therapeutic efficacy against human vascular diseases.
\end{abstract}

Keywords: Umbilical cord blood; Mesenchymal stem cells; Endothelial cell; Angiogenesis; Egr-3

Abbreviations: Ab: Antibody; AAD: 7-Amino-Actinomycin D; BCA: Bicinchoninic Acid Assay; Cal C: Calphostin C; CsA: Cyclosporine A; DMSO: Dimethyl Sulfoxide; Egr-3: Early Growth Response 3; ERK: Extracellular-signal Related Kinase; FBS: Fetal Bovine Serum; GAPDH: Glyceraldehyde-3-phosphate Dehydrogenase; HUVEC: Human Umbilical Vein Endothelial Cell; ISO: Isoproterenol; MAPK: Mitogen-activated Protein Kinase; MSC: Mesenchymal Stem Cell; PBS: Phosphate-buffered Saline; PKC: Protein Kinase C; PMA: Phorbol-12-myristate-13-acetate; RT: Room Temperature; SDF-1: Stromal Cell-derived Factor 1; SDS-PAGE: Sodium Dodecyl Sulphatepolyacrylamide Gel Electrophoresis; siRNA: Small Inhibitory RNA; SD: Standard Deviation; UCB: Umbilical Cord Blood; VEGF: Vascular Endothelial Growth Factor

\section{Introduction}

In order to maintain homeostasis, tissues of multicellular organisms are endowed with a vascular system responsible for gas and nutrient exchange. Maintenance of this paramount function is dependent on endothelium integrity. Our view of the endothelium has changed gradually over the years. Whereas the endothelium used to be considered a simple inert barrier between blood vessels and subjacent tissues, it is now recognized as a specialized layer of cells - endothelial cells (ECs) - [1]. It is clear that ECs have critical roles in organism homeostasis and in moving nutrients, gases and waste products to and from cells [2-4]. Therefore, loss of vascular endothelial homeostasis critically contributes to the origin and/or progression of numerous ischemic, inflammatory, infectious and immune diseases in humans [5].

There is compelling evidence that vascular maintenance arises due mainly to two non-exclusive mechanisms: local maintenance by pre-existing or mature (terminally differentiated) ECs (sprouting angiogenesis) [6] and distant maintenance by specific recruitment of vascular precursors from bone marrow (vasculogenesis) [7]. A variety of both cellular and extracellular molecules, including numerous growth and early response factors, chemokines, interleukins and metalloproteinases, take part in active angiogenesis [8,9]. These molecules include the early growth response factor (Egr)-3, which is a transcription factor rapidly induced by extracellular stimuli that regulates proliferation and differentiation in multiple cell types such as ECs [10]. Moreover, Egr-3 has been reported as one of the most strongly activated genes by vascular endothelial growth factor (VEGF) and its specific suppression attenuates EC growth $[11,12]$. At the molecular level, Egr-3 has been related to intracellular signaling mediated by protein kinase $\mathrm{C}$ (PKC) $[13,14]$ and mitogen-activated protein kinase (MAPK)/extracellular-signal related kinase (ERK) $[15,16]$. Notably, Li

*Corresponding authors: Santiago Roura, ICREC Research Program, IGTP Crta. Canyet, s/n, 08916 Badalona, Barcelona, Spain, Tel: +34-93-4978663; Fax: +34-93-4978654; E-mail: sroura@gmail.com; sroura@igtp.cat

Antoni Bayes-Genis, Full Professor, Department of Medicine, UAB Barcelona, Spain, Tel: +34-93-4973743; Fax: +34-93-4978654; E-mail: abayes.germanstrias@gencat.cat

\section{Received May 29, 2013; Accepted June 27, 2013; Published June 29, 2013}

Citation: Roura S, Bagó JR, Gálvez-Montón C, Blanco J, Bayes-Genis A (2013) In Vitro Characterization of the Molecular Machinery Regulating Umbilical Cord Blood Mesenchymal Stem Cell Angiogenesis: A Step Towards Multipoten Stem Cell Therapy for Vascular Regeneration. J Stem Cell Res Ther 3: 140. doi:10.4172/2157-7633.1000140

Copyright: (C) 2013 Roura S, et al. This is an open-access article distributed unde the terms of the Creative Commons Attribution License, which permits unrestricted use, distribution, and reproduction in any medium, provided the original author and source are credited. 
Citation: Roura S, Bagó JR, Gálvez-Montón C, Blanco J, Bayes-Genis A (2013) In Vitro Characterization of the Molecular Machinery Regulating Umbilical Cord Blood Mesenchymal Stem Cell Angiogenesis: A Step Towards Multipotent Stem Cell Therapy for Vascular Regeneration. J Stem Cell Res Ther 3: 140. doi:10.4172/2157-7633.1000140

Page 2 of 10

et al. [15] described Egr-3 as the precise effector molecule of MAPKERK signaling involved in enduring memory formation in activated neurons.

In recent years, umbilical cord blood (UCB) has emerged as a promising source of cells for human regenerative medicine [17] and UCB-derived mesenchymal stem cells (MSCs) have been shown to promote vascular growth in vivo [18]. However, further understanding of the molecular mechanisms regulating UCBMSC angiogenesis should provide insights that may help develop more effective therapies against human diseases that involve vascular deficits. Particularly, we hypothesized that Egr-3 was a key mediator of UCBMSC angiogenesis because its expression increased as cells gained EC characteristics and self-organized to form branched polygonal cell networks similar to those developed by bona fide ECs [18].

Thus, with the overarching aim of improving their therapeutic efficacy, we here examined the intracellular regulatory machinery involved in the angiogenic behaviour exhibited by UCBMSCs by: (1) exposure to known modulators of both Egr-3 and EC angiogenesis; (2) assessment in the standard Matrigel assay; and (3) specific suppression of Egr-3 expression using small interfering RNA (siRNA) technology.

\section{Materials and Methods}

\section{Cell culture and treatments}

Isolation and culture of UCBMSCs were previously described in detail $[14,18,19]$. Briefly, a total of 25 UCB samples $(60-100 \mathrm{ml})$ from the umbilical cord vein were processed $12 \mathrm{~h}$ post-extraction. Blood cells were clarified by previous centrifugation and resuspended in $30 \mathrm{ml}$ calcium- and magnesium-free phosphate-buffered saline (PBS) (Invitrogen). The cell suspension layered over $1.077 \mathrm{~g} / \mathrm{ml}$ Lymphoprep (Nycomed) was centrifuged at $400 \mathrm{~g}$ for 30 minutes. Mononuclear cells recovered by centrifugation were eliminated from contaminant red blood cells by incubation with lysis reagent PharmLyse (BD Biosciences) for 15 minutes. Recovered cells were grown in a-MEM supplemented with $30 \%$ fetal bovine serum (FBS), $1 \%$ penicillin-streptomycin (Invitrogen), $1 \mathrm{mM} \mathrm{L}$-glutamine and $10^{-7} \mathrm{M}$ dexamethasone (Sigma) at $37^{\circ} \mathrm{C}$ in $5 \% \mathrm{CO}_{2}$ in air. Adherent cells were pooled by trypsinization, designated as primary culture (passage 0 ) and replated for further expansion in the same medium supplemented with $10 \%$ FBS and without dexamethasone.

When indicated, cells were cultured in a-MEM with $2 \%$ FBS plus: phorbol-12-myristate-13-acetate (PMA) (100 nM, $2 \mathrm{~h}$ ), dimethyl sulfoxide (DMSO) $(0.2 \% \mathrm{v} / \mathrm{v}, 16 \mathrm{~h})$ and CsA $(200 \mathrm{ng} / \mathrm{ml}, 16 \mathrm{~h})$ (Sigma); U0126 $(20 \mu \mathrm{M}, 16 \mathrm{~h}), \mathrm{U} 0124(20 \mu \mathrm{M}, 16 \mathrm{~h})$ and Cal C $(1 \mu \mathrm{M}, 16 \mathrm{~h})$ (Calbiochem); ISO ( $1 \mu \mathrm{M}, 16 \mathrm{~h}$; Boehringer Ingelheim); and human recombinant VEGF (10 ng/ml, 48 h; R\&D Systems).

The study protocol was approved by the local ethics committee (Comitè Ėtic d'Investigació Clínica, HuGTiP, Ref. CEIC: EO-12022) and conformed to the principles outlined in the Declaration of Helsinki. In all cases written informed consent was obtained from the donors.

\section{Flow cytometry}

To measure expression of surface antigens, cells $\left(5 \times 10^{5} / 100 \mu \mathrm{l}\right)$ were labelled with $10 \mu \mathrm{l}$ of mouse fluorescein (FITC)- or phycoerythrin (PE)-conjugated antibodies (Abs) against human CD105 (Serotec), CD90, CD14, CD34 and CD45 (BD Pharmingen) during $30 \mathrm{~min}$ at room temperature (RT). Labelled isotype-matched IgG (Caltag Laboratories) were used as negative controls. Intensity levels for each antigen were calculated as the ratio between specific antibody and control ( $1=$ no difference). Data acquisition and further analysis were carried out using a Coulter EPICS XL flow cytometer and the Expo32 software (Beckman Coulter), respectively.

Changes in cell viability were also assessed by flow cytometry using the Annexin V Apoptosis Detection Kit and the 7-amino-actinomycin $\mathrm{D}$ (AAD) viability staining solution (eBiosciences). Cells $\left(5 \times 10^{5}\right)$ were labelled according to the supplier's instructions. Data acquisition and analysis were then carried out using a Coulter EPICS XL flow cytometer and the Expo32 software (Beckman Coulter), respectively. As positive control for the induction of apoptosis, cells were treated with camptothecin $(10 \mu \mathrm{M}, 16 \mathrm{~h}$; Sigma $)$.

\section{Differentiation assays}

Adipogenic induction: UCBMSCs were cultured in a-MEM (Sigma) supplemented with $10 \% \mathrm{FBS}, 1 \mathrm{mM} \mathrm{L}$-glutamine and $1 \%$ penicillin/streptomycin, $1 \mu \mathrm{M}$ dexamethasone, $0.5 \mu \mathrm{mM}$ isobutylmethylxanthine, $10 \mu \mathrm{g} / \mathrm{ml}$ insulin and $100 \mu \mathrm{M}$ indomethacin (Sigma). Medium was replaced every 3-4 days and differentiated cells were detected at day 14 following Oil red O (Sigma) staining, which detects the presence of intracellular lipid accumulation.

Osteogenic induction: UCBMSCs were cultured in a-MEM supplemented with $10 \%$ FBS, $1 \mathrm{mM}$ L-glutamine and $1 \%$ penicillin/streptomycin, $100 \mathrm{nM}$ dexamethasone, $10 \mathrm{mM}$ sodium $\beta$-glycerophosphate and $0.05 \mathrm{mM}$ ascorbic acid during 14 days. Deposition of calcium matrix was then detected by specific staining with Alizarin red S (Sigma).

\section{Quantitative RT-PCR}

Whole RNA content was isolated from cells using the QuickPrep Extraction Kit (Amersham). cDNA from $2 \mu \mathrm{g}$ of RNA was obtained using random hexamers and the Script ${ }^{\mathrm{TM}}$ One-Step RT-PCR Kit (BioRad) following the supplier's protocol. Subsequently, $2 \mu$ of cDNA were amplified in a final volume of $50 \mu \mathrm{l}$ containing $25 \mu \mathrm{l}$ TaqMan $2 \mathrm{X}$ Universal PCR Master Mix and $2 \mu \mathrm{l}$ of the following FAM-labelled primer/probes (Applied Biosystems): CD31 (Hs00169777_m1), CD34 (Hs00990732_m1), CD36 (Hs00169627_m1), CD102 (Hs00168384_ $\mathrm{m} 1$ ), early growth response factor (Egr)-3 (Hs00231780_m1), stromal cell-derived factor (SDF)-1 $\alpha$ (Hs00171022_m1), VEGF (Hs00173626_ $\mathrm{m} 1$ ), sarcomeric $\alpha$-actinin (Hs00241650_m1) and glyceraldehyde-3phosphate dehydrogenase (GAPDH) (Hs99999905_m1). Each sample was run in duplicate in the same experiment and amplification data from three independent experiments were analyzed on the ABI Prism 7000 Sequence Detection System. The $\Delta$ threshold cycle (Ct) method was used to quantify the relative expression for each gene using GAPDH as endogenous reference [20].

\section{Indirect immunofluorescence}

Egr-3 protein expression was analyzed following fixation with $4 \%$ paraformaldehyde (Sigma) for $10 \mathrm{~min}$ at room temperature (RT). Cells were permeabilized with magnesium-free PBS supplemented with $10 \%$ horse serum and $0.5 \%$ Triton X-100 (Sigma) during $2 \mathrm{~h}$ at RT. A specific rabbit Ab against human Egr-3 ( $2 \mu \mathrm{g} / \mathrm{ml}$; Santa Cruz Biotechnology) was then applied. Cell nuclei were also counterstained with Hoechst $(0.5 \mu \mathrm{g} / \mathrm{ml})$ (Sigma) for $10 \mathrm{~min}$ at RT. A donkey anti-rabbit Alexa Fluor 488-conjugated $\mathrm{Ab}(1 \mu \mathrm{g} / \mathrm{ml}$; Invitrogen) was finally used to detect labelled cells under a Leica confocal laser scanning microscope (TCS SP5).

\section{Western blotting}

Whole cell extracts were obtained using the Mammalian Cell 
Citation: Roura S, Bagó JR, Gálvez-Montón C, Blanco J, Bayes-Genis A (2013) In Vitro Characterization of the Molecular Machinery Regulating Umbilical Cord Blood Mesenchymal Stem Cell Angiogenesis: A Step Towards Multipotent Stem Cell Therapy for Vascular Regeneration. J Stem Cell Res Ther 3: 140. doi:10.4172/2157-7633.1000140

Lysis kit (Sigma) according to manufacturer's instructions. Equivalent amounts of protein (assayed with the BCA protein assay; Pierce Biotechnology) were fractionated by $10 \%-15 \%$ SDS-PAGE and transferred to nitrocellulose membranes $(0.45 \mu \mathrm{m}$ pore size; Bio-Rad). Membranes were blocked with PBS supplemented with 5\% non-fat dry milk and $0.05 \%$ Tween $-20 \mathrm{o} / \mathrm{n}$ at $4{ }^{\circ} \mathrm{C}$, and probed with mouse antihuman Abs against $\beta$-actin (1/1000 dilution), ERK1/2 $(0.5 \mu \mathrm{g} / \mathrm{ml})$ and phospho (p)-ERK ( $1 \mu \mathrm{g} / \mathrm{ml})$ (Santa Cruz Biotechnology). Protein bands were detected using a HRP-conjugated goat anti-mouse IgG $(\mathrm{H}+\mathrm{L}) \mathrm{Ab}(1 / 50000$ dilution; Pierce Biotechnology), and visualized using the Super Signal West Pico Chemiluminiscent Substrate (Pierce Biotechnology) and the Bio-Rad Molecular Imager Chemidoc ${ }^{\mathrm{TM}}$ XRS+. Protein levels were quantified using the Quantity one 1-D Analysis software (Bio-Rad) and expressed as arbitrary units of optical density. Background signals were subtracted from a membrane region close to the bands of interest. Densitometric p-ERK values were normalized to $\beta$-actin (to control for variation in protein loading) and to total ERK.

\section{Matrigel assay}

The capacity to form cell networks was assessed using the In vitro Angiogenesis Assay Kit (Chemicon) as previously described [18]. Briefly, control UCBMSCs, which had been either pre-treated or transduced with siRNA sequences, were detached using nonenzymatic cell dissociation medium (Sigma) to avoid cell membrane antigen proteolysis. Cells $\left(1 \times 10^{5}\right)$ were then seeded onto 24 -well plates pre-coated with $200 \mu$ l ECMatrix (Matrigel). Agonists and selective inhibitors added to the cultures were kept in the samples for the duration of the experiment. The cell networks that developed were examined from 10 images. Angiogenic capacity was measured as the number of network circles per $\mathrm{mm}^{2}$, a measurement equivalent to the classic determination of total network length [21]. Three independent cultures were tested per condition.

\section{siRNA transfection}

Cells were transduced with $2 \mu \mathrm{g}$ siControl-GFP, siControl ${ }_{\mathrm{A}}$ and siEgr-3 (Santa Cruz Biotechnology). The siEgr-3 consisted of a pool of 3-5 target-selective 19-25 nucleotides in length designed to efficiently knockdown Egr-3 gene expression. All cell transductions were performed via a nonviral Nucleofector ${ }^{\circledR}$ procedure (Amaxa ${ }^{\circledR}$ Cell Line Optimization Nucleofector ${ }^{\circledR}$ Kit; Lonza Cologne AG) with the program U23 for transfection of human MSCs according to the manufacturer's instructions [22].

\section{Statistical analysis}

Statistical analysis was performed using two-tailed Student's $t$-tests. Values are expressed as mean \pm standard deviation (SD). Oneway analysis of variance with Tukey's B post-hoc analysis was applied to determine significance among more than two groups. Descriptive statistics were performed using SPSS Statistics 15.0.1 (SPSS Inc.), and statistical tests were considered significant when $P<0.05$.

\section{Results}

\section{Modulation of Egr-3 expression by PKC/MAPK/ERK in UCBMSCs}

Primary cultures of elongated fibroblast-like cells were established from human UCB samples. Cultured cells were homogenously recognized as MSCs according to the International Society for Cellular Therapy criteria (standard surface antigen pattern and multipotency) [23]. The cells were strongly positive for CD105 (18.5 \pm 0.14$)$ and CD90
$(60 \pm 11)$, and consistently negative for CD45 $(1 \pm 0)$, CD34 (1.2 \pm 0.2$)$ and CD14 $(1 \pm 0)$ (Figure 1A). Induction of UCBMSCs with adipogenic and osteogenic media resulted in intracellular accumulation of lipid droplets and in high extracellular deposition of calcium, respectively (Figure 1B).

We had previously demonstrated the following: 1) Egr-3 expression was highly promoted when human UCBMSCs differentiated towards the EC lineage; 2) in Matrigel, similar to mature ECs such as human umbilical vein ECs (HUVECs), UCBMSCs had the ability to rapidly migrate developing well-organized vascular-like networks; and 3) developed networks showed high amounts of Egr-3 within the nuclei of aligned cells [18].

In accordance with this background, we further investigated the molecular machinery regulating the angiogenic behaviour of UCBMSCs and whether Egr- 3 was mechanistically involved. Thus, cells were then exposed to PMA, CsA and VEGF, known modulators of Egr-3 expression and EC angiogenesis, and seeded in Matrigel. In this standard assay of angiogenesis, which is often preferred over other in vitro assays because of its ease of use, rapidity and the ability to measure key steps in angiogenesis, cells rearrange and align forming a polygonal cell network resembling growing blood vessels from which angiogenic capacity may be evaluated, e.g. by measuring the number of network circles per unit area [24-26]. Egr-3 gene expression level was also assessed using real-time PCR, which is the most direct, sensitive, reproducible and accurate method to quantify gene activity [27].

While PMA induced increases in Egr-3 mRNA and protein and promoted the capacity to form well-organized vascular-like networks in comparison with untreated UCBMSCs $(P<0.001)$, the addition of neither CsA nor VEGF significantly affected any of these properties (Figure 2). In addition, exposure to calphostin $\mathrm{C}$ (Cal C), which is a commonly-used inhibitor of $\mathrm{PKCa}$, drastically reduced both PMA-induced Egr-3 gene transcription $(P<0.001)$ and the Matrigelmediated formation of cell network circles by UCBMSCs $(P<0.001$; Figure 2). Stimulation of cells with the $G$ protein-coupled receptor agonist isoproterenol (ISO) [28] also doubled Egr-3 gene expression in comparison with untreated cells $(P=0.013$; data not shown). In contrast to the effect of each agent individually, combined ISO and PMA treatment had a reduced effect in comparison with PMA alone regarding Egr-3 activation and network-forming capacity (Figure 2). U0126, which is a selective pharmacological inhibitor of MAPK/ ERK signaling [29], suppressed both Egr-3 transcriptional activation and cell network organization in treated cells $(P=0.015$ and $P=0.016$, respectively; Figure 2). Moreover, the presence of $\mathrm{Cal} \mathrm{C}$ in the cell culture medium significantly reduced PMA-induced angiogenesis as well as $E g r-3$ activation in UCBMSCs $(P<0.001$; Figure 2$)$. Neither cell network-forming ability nor Egr-3 expression in UCBMSCs were affected by addition of U0124, an inactive structural analogue of U0126 (Figure 2).

Importantly, analysis of both Annexin $\mathrm{V}$ expression and 7-aminoactinomycin D (7-AAD) staining by flow cytometry showed that the observed changes in the angiogenic behaviour of UCBMSCs did not result from variations in cell apoptotic rate and/or viability following each cell treatment (Figure 3). Western blot experiments also evidenced that the addition of PMA to UCBMSCs induced an increase in ERK1/2 phosphorylation $(P=0.021)$, and that this effect was significantly abolished by treatment with U0126 $(P=0.014$; Figure 4$)$.

Collectively, these observations suggest so far that the modulation of Egr-3 expression and the promotion of network-forming capacity in UCBMSCs are mediated through PKC/MAPK/ERK signaling. 
Citation: Roura S, Bagó JR, Gálvez-Montón C, Blanco J, Bayes-Genis A (2013) In Vitro Characterization of the Molecular Machinery Regulating Umbilical Cord Blood Mesenchymal Stem Cell Angiogenesis: A Step Towards Multipotent Stem Cell Therapy for Vascular Regeneration. J Stem Cell Res Ther 3: 140. doi:10.4172/2157-7633.1000140

\section{A}

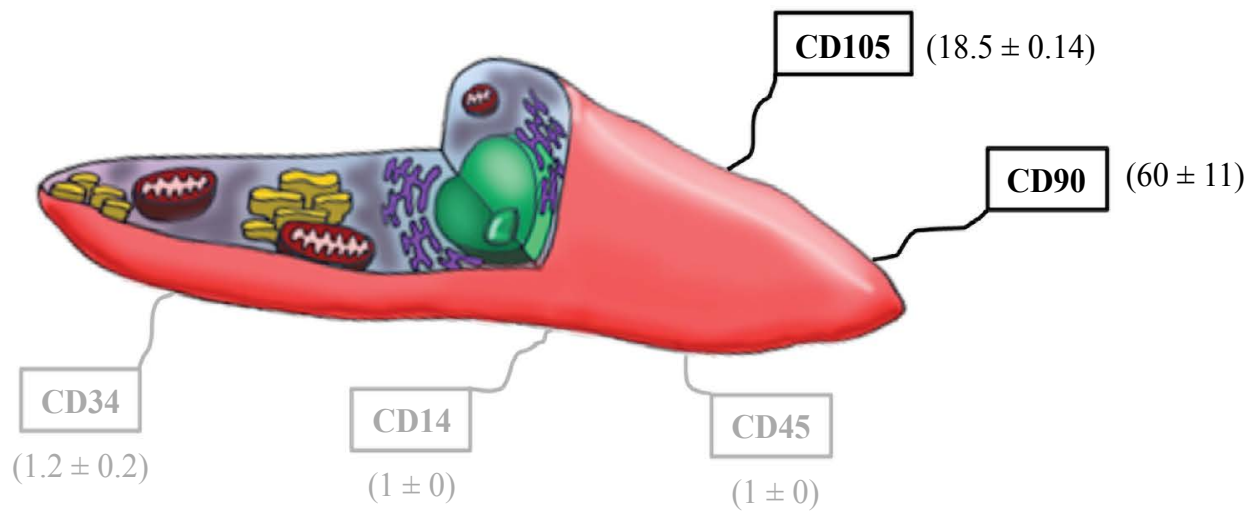

B

Multipotency

(lineage-specific differentiation)
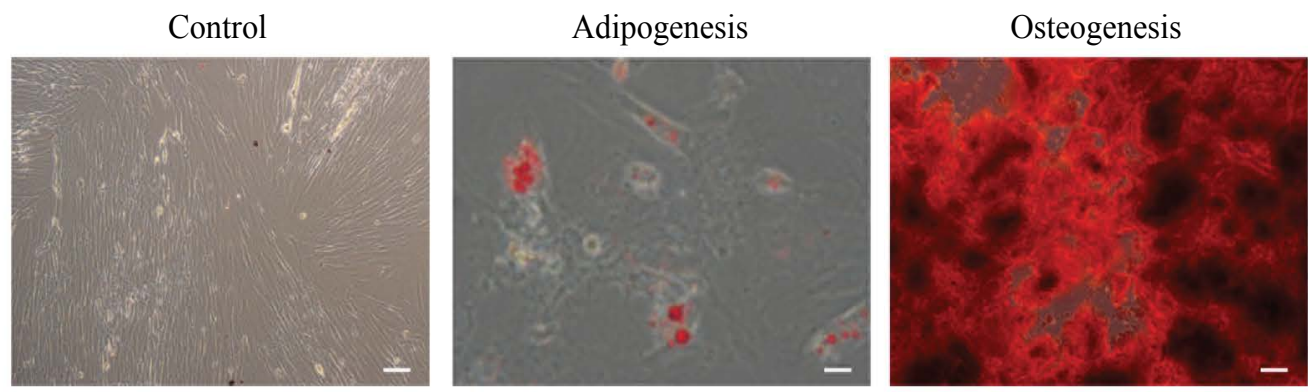

Figure 1: Characterization of primary cultures of human UCBMSCs. A) Schematic illustration of an UCBMSC showing fluorescence intensity data in arbritary units from surface antigen expression analysis by flow cytometry. UCBMSCs were strongly positive for CD105 and CD90 (60 \pm 11 ), and consistently negative for CD45, CD34, and CD14. Values are expressed as mean \pm SD. B) Standard MSC differentiation into adipogenic and osteogenic cell-lineages. Images display a control, nondifferentiated (left) and differentiated cell cultures following staining with Oil red $\mathrm{O}$ (middle) and Alizarin red $\mathrm{S}$ (right). Scale bars: $100 \mu \mathrm{m}$.

\section{Specific suppression of Egr-3 abrogates cell network capacity of UCBMSCs}

To clearly demonstrate that Egr-3 directly regulates UCBMSC angiogenic behaviour, cells were transduced with a specific siRNA against Egr-3. This strategy resulted in a marked reduction of both Egr3 mRNA and protein levels in siEgr-3-transduced cells relative to those transduced with control siRNA ( siControl $\left._{A}\right)(P<0.001$; Figures $5 \mathrm{~A}$ and $5 \mathrm{~B})$. Moreover, the number of developed network circles was inhibited by $85 \%$ in siEgr-3-transduced UCBMSCs (Figure $5 \mathrm{~B}$ ). Interestingly, specific suppression of Egr-3 expression had no effect on either cell apoptotic rate or cell viability (Figure 3 ). Data from quantitative RTPCR analysis of siEgr-3-transduced UCBMSCs finally indicated that the expression pattern of EC markers was not broadly different in comparison with that exhibited by siControl -transduced cells. Only CD36 expression was reduced by $50 \%$, whereas CD102 activation increased (2-fold) in siEgr-3-transduced cells (Figure 5C). Thus, the transcriptional profile observed in siEgr-3-transduced UCBMSCs is similar to that reported by Suehiro et al. [10] who revealed a preferential role for Egr-3 in regulating mainly genes involved in cell adhesion and migration (CD102), growth, and homeostasis (CD36) instead of EC fate acquisition.

\section{Discussion}

The presented results identify Egr-3, which is a transcriptional factor highly expressed during mature, terminally differentiated EC angiogenesis $[11,30]$, as a key mediator of UCBMSC angiogenesis. In particular, we find that a central PKCa/MAPK/ERK pathway common to mature ECs regulates Egr-3 in UCBMSCs. Moreover, selective suppression of Egr-3 significantly abrogates the angiogenic capacity of UCBMSCs. Following Egr-3 inhibition, the reduction in network circles developed by UCBMSCs was similar to that previously found in ECs [10]. Thus, our findings suggest the existence of a conserved regulatory machinery regulating angiogenesis between ECs and multipotent MSCs (Figure 6). This knowledge should provide insights that may help develop more effective therapies against human vascular diseases.

In addition, our results agree with the traditional concept that PKC has an essential role in angiogenesis, based on the findings that 
Citation: Roura S, Bagó JR, Gálvez-Montón C, Blanco J, Bayes-Genis A (2013) In Vitro Characterization of the Molecular Machinery Regulating Umbilical Cord Blood Mesenchymal Stem Cell Angiogenesis: A Step Towards Multipotent Stem Cell Therapy for Vascular Regeneration. J Stem Cell Res Ther 3: 140. doi:10.4172/2157-7633.1000140
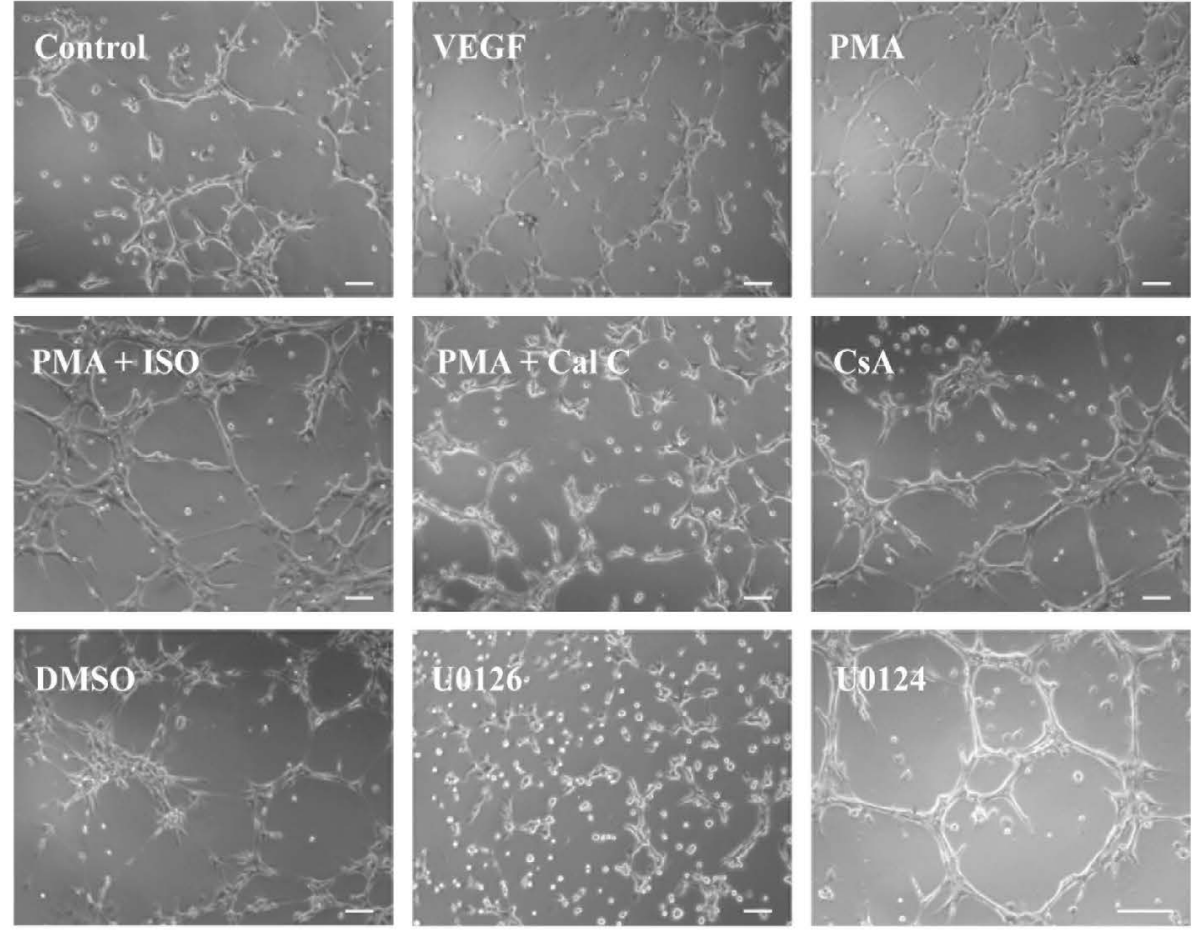

\section{Egr-3}

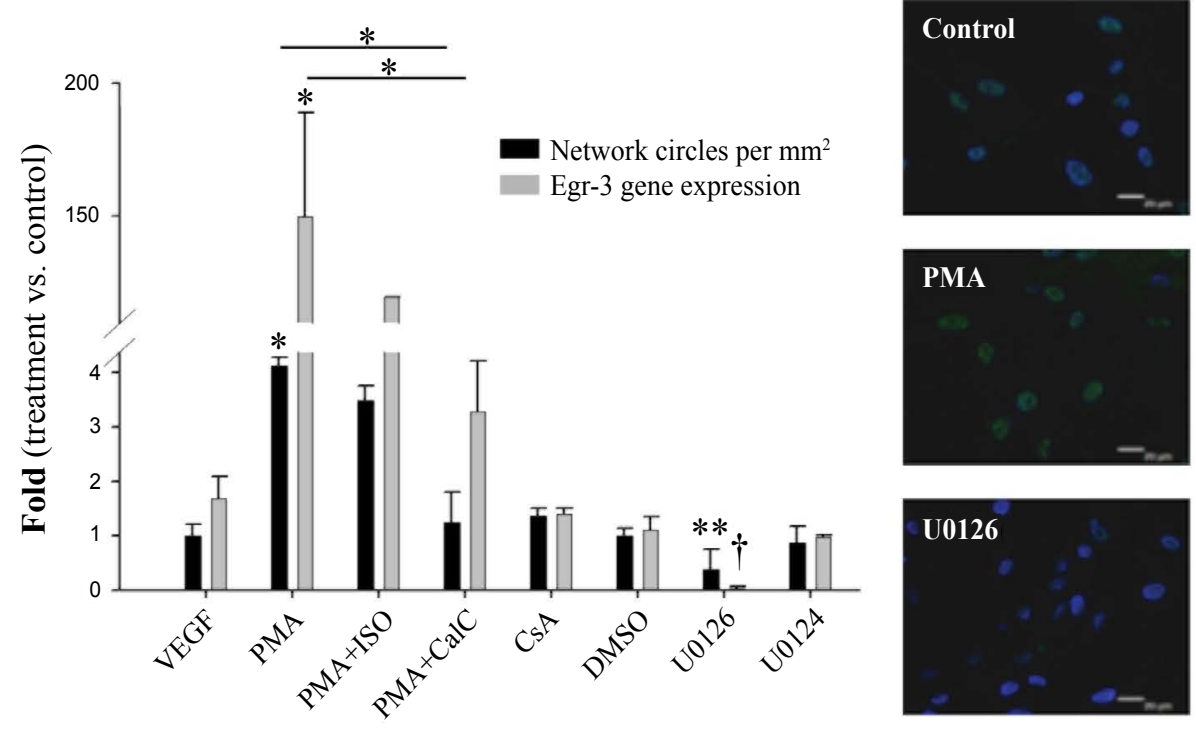

Figure 2: Modulation of Egr-3 expression levels and angiogenic capacity by UCBMSCs. Representative images and quantification of the cell network-forming capacity, measured as the number of network circles per unit area, of UCBMSCs after $2 \mathrm{~h}$ in Matrigel following treatment with a specific agonist (PMA) and inhibitors (Cal C and U0126) of PKCa/MEK/Egr-3 signaling. Scale bars: $100 \mu \mathrm{m}$. Histogram represents the relative levels of Egr-3 gene expression and cell network formation compared with those in control or untreated cells. DMSO and U0124 were used as vehicle and negative control, respectively. Note that there is equivalence in the scales of gene expression and network circles $/ \mathrm{mm}^{2}$, maybe indicating the close relationship between these two cellular events. Data are from three independent experiments and all values are expressed as mean \pm SD. Specific detection of Egr-3 protein in control, PMA- and U0126-treated UCBMSC cultures by indirect immunofluorescence. ${ }^{*} P<0.001,{ }^{*} P=0.016$ and ${ }^{\dagger} P=0.015$. Scale bars: $20 \mu \mathrm{m}$. A minimum of 10 microscopic fields per condition and experiment ( $\mathrm{n}=3$ ) were analyzed with similar results.

phorbol esters, by mimicking the natural PKC activator diacylglycerol, induce angiogenesis [31-33] and that specific knockdown of PKC prevents PMA-induced angiogenesis [13]. In our experiments, Egr-3 expression and cell network-forming capacity of UCBMSCs was not affected by VEGF levels or by the powerful immunosuppressive agent CsA, which are known to modulate VEGF-mediated EC angiogenesis and Egr-3 levels in hematopoietic-lineage cells such as lymphocytes $\mathrm{T}$ respectively [11,13,30,34-37]. In regards to both VEGF and CsA, the 
Citation: Roura S, Bagó JR, Gálvez-Montón C, Blanco J, Bayes-Genis A (2013) In Vitro Characterization of the Molecular Machinery Regulating Umbilical Cord Blood Mesenchymal Stem Cell Angiogenesis: A Step Towards Multipotent Stem Cell Therapy for Vascular Regeneration. J Stem Cell Res Ther 3: 140. doi:10.4172/2157-7633.1000140
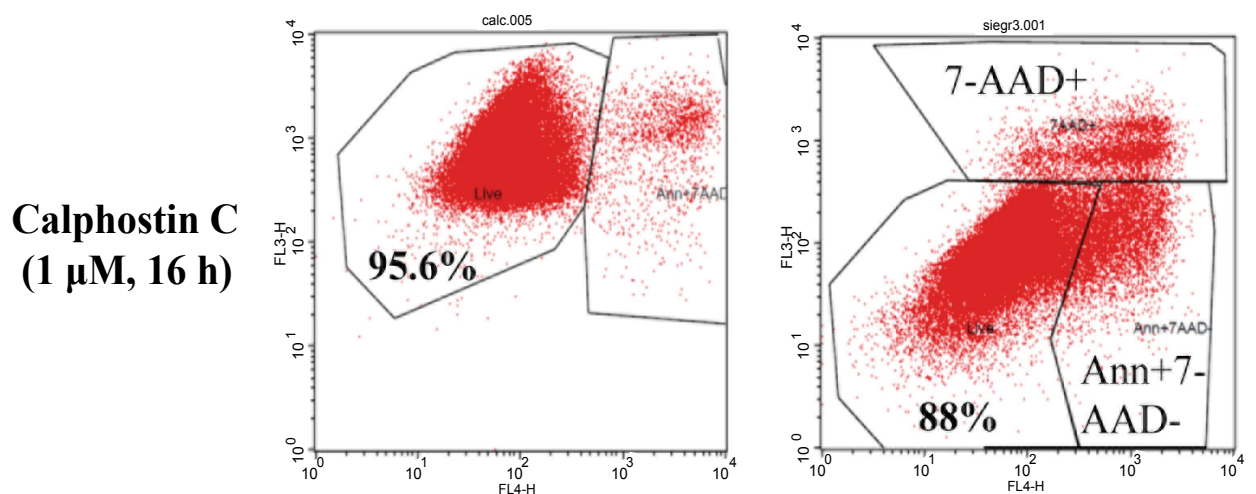

\section{siEgr-3}

U0126

$(20 \mu \mathrm{m}, 16 \mathrm{~h})$
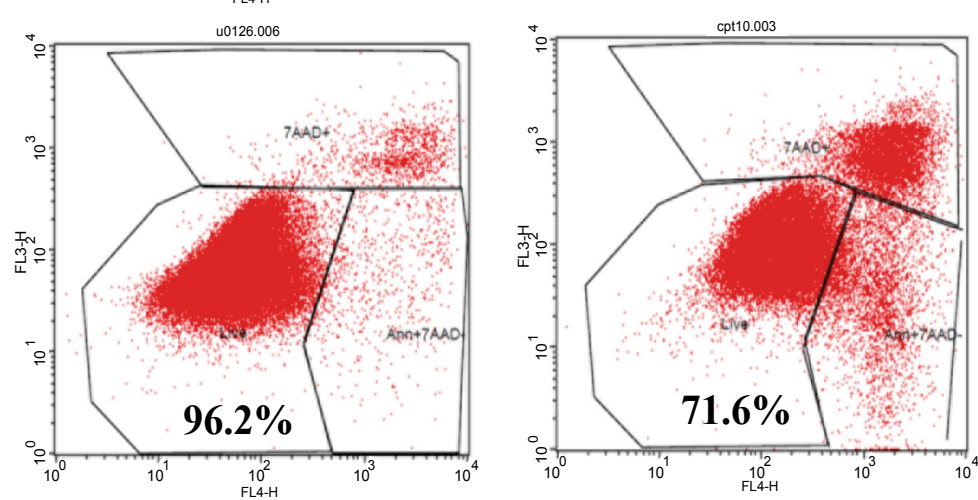

\section{Camptothecin}

$(10 \mu \mathrm{M}, 16 \mathrm{~h})$

Figure 3: Analysis of cell viability. Raw data from representative analysis of Annexin (Ann) V expression and 7-AAD staining in UCBMSCs treated with camptothecin, used as positive control for cell apoptosis, calphostin C and U0126, and following transduction with siEgr-3. Ann+7-AAD- and 7-AAD+ label early and late apoptotic cells, respectively. The percentage of live cells under each condition is indicated in the corresponding dot plot. Treatment with camptothecin induced a moderate amount of programmed cell death, indicative of intrinsic UCBMSC robustness. The 10-fold increase in the fluorescence emitted by calphostin C-treated cells did no effect measured levels of cell death. $\mathrm{N}=2$ for each experimental condition with similar results.
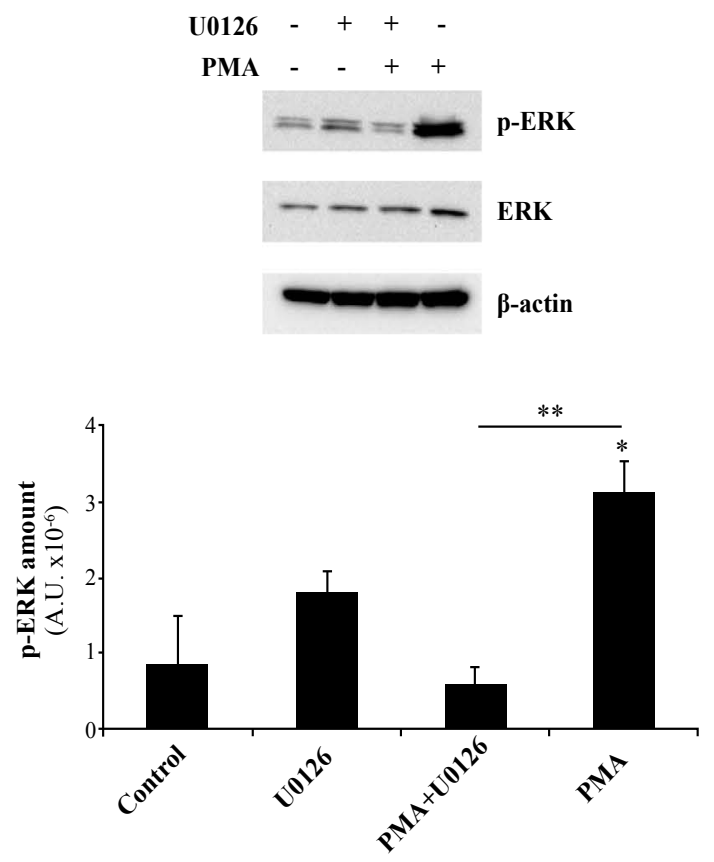

Figure 4: PMA induces phosphorylation of ERK1/2 in UCBMSCs. Western blot panels show that ERK1 (44 kDa) and ERK2 (42 kDa) were phosphorylated following exposure to PMA. In contrast, addition of U0126 suppressed PMA-mediated ERK1/2 phosphorylation. Histogram depicts protein levels as arbitrary units (A.U.), which were determined by densitometry in three independent experiments. A 3.6-fold activation of ERK1/2 was detected in PMA-treated relative to control cells. $\mathrm{N}=3$ for each experimental condition with similar results. Values are expressed as mean $\pm \mathrm{SD}$. ${ }^{*} P=0.021$ and ${ }^{* \star} P=0.014$. 
Citation: Roura S, Bagó JR, Gálvez-Montón C, Blanco J, Bayes-Genis A (2013) In Vitro Characterization of the Molecular Machinery Regulating Umbilical Cord Blood Mesenchymal Stem Cell Angiogenesis: A Step Towards Multipotent Stem Cell Therapy for Vascular Regeneration. J Stem Cell Res Ther 3: 140. doi:10.4172/2157-7633.1000140

\section{A}

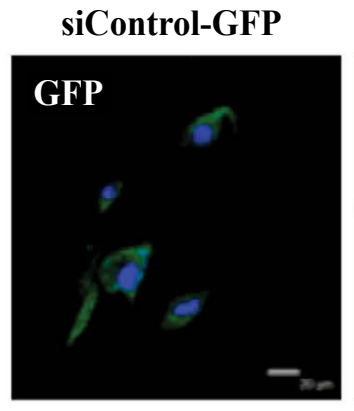

siControl $_{A}$

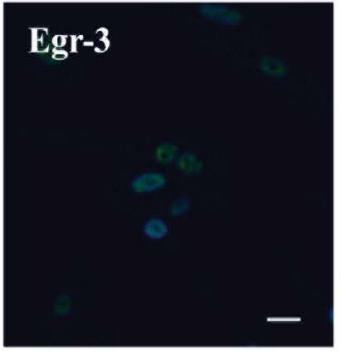

\section{siEgr-3}

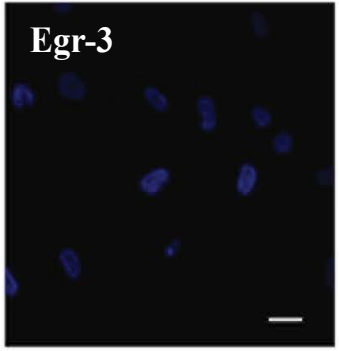

B
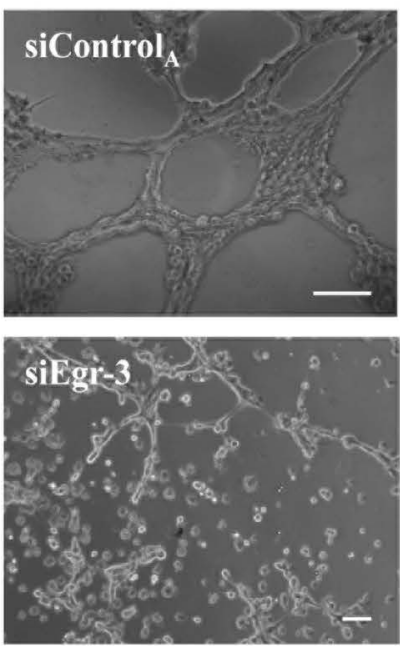

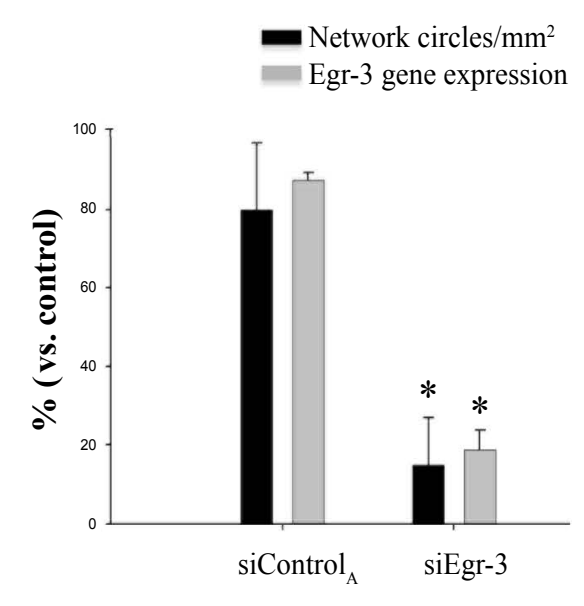

Network circles $/ \mathrm{mm}^{2}$

\section{siEgr-3}

C

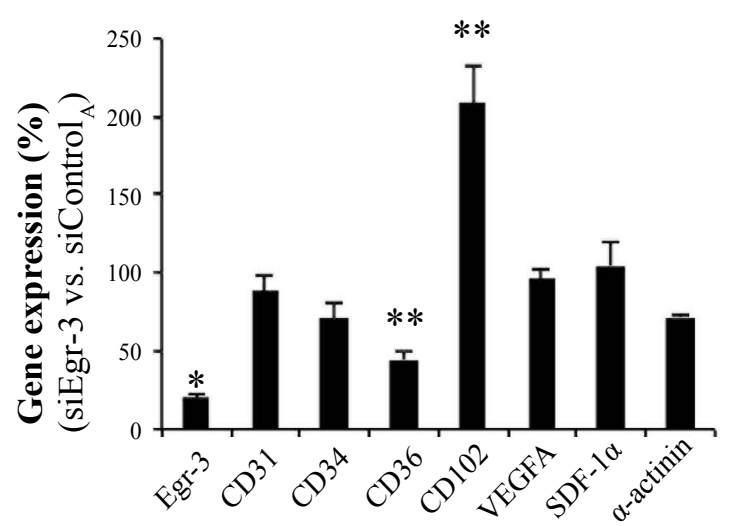

Figure 5 Inhibition of UCBMSC angiogenic capacity by specific suppression of Egr-3. (A) Representative images showing internalization of control sequence (siControl-GFP conjugate) (left), and specific detection of Egr-3 in siControl - (middle) and siEgr3-transduced (right) cells. Scale bars: 20 um (B) Representative images of the cell network developed by UCBMSCs transduced with siControl and siEgr-3 after $5 \mathrm{~h}$ in Matrigel. Histogram indicates the number of network circles and the transcriptional levels of Egr-3 in siControl ${ }_{A}$ - and siEgr3-transduced cells. A minimum of 10 microscopic fields per condition and experiment ( $n=3$ ) were analyzed. Scale bars: $100 \mu \mathrm{m}$. Values are expressed as mean \pm SD and ${ }^{*} P<0.001$. (C) Transcriptional profile exhibited by siEgr-3-transduced UCBMSCs showing the relative changes (compared with siControl -transduced cells) in specific (CD31, CD34, CD36, CD102 and VEGFA) and non-specific (SDF-1 $\alpha$ and sarcomeric $\alpha$-actinin) EC genes. $\mathrm{N}=3$ with similar results. Values are expressed as mean $\pm \mathrm{SD}$. ${ }^{*} P<0.001$ and ${ }^{* *} P<0.05$. 
Citation: Roura S, Bagó JR, Gálvez-Montón C, Blanco J, Bayes-Genis A (2013) In Vitro Characterization of the Molecular Machinery Regulating Umbilical Cord Blood Mesenchymal Stem Cell Angiogenesis: A Step Towards Multipotent Stem Cell Therapy for Vascular Regeneration. J Stem Cell Res Ther 3: 140. doi:10.4172/2157-7633.1000140

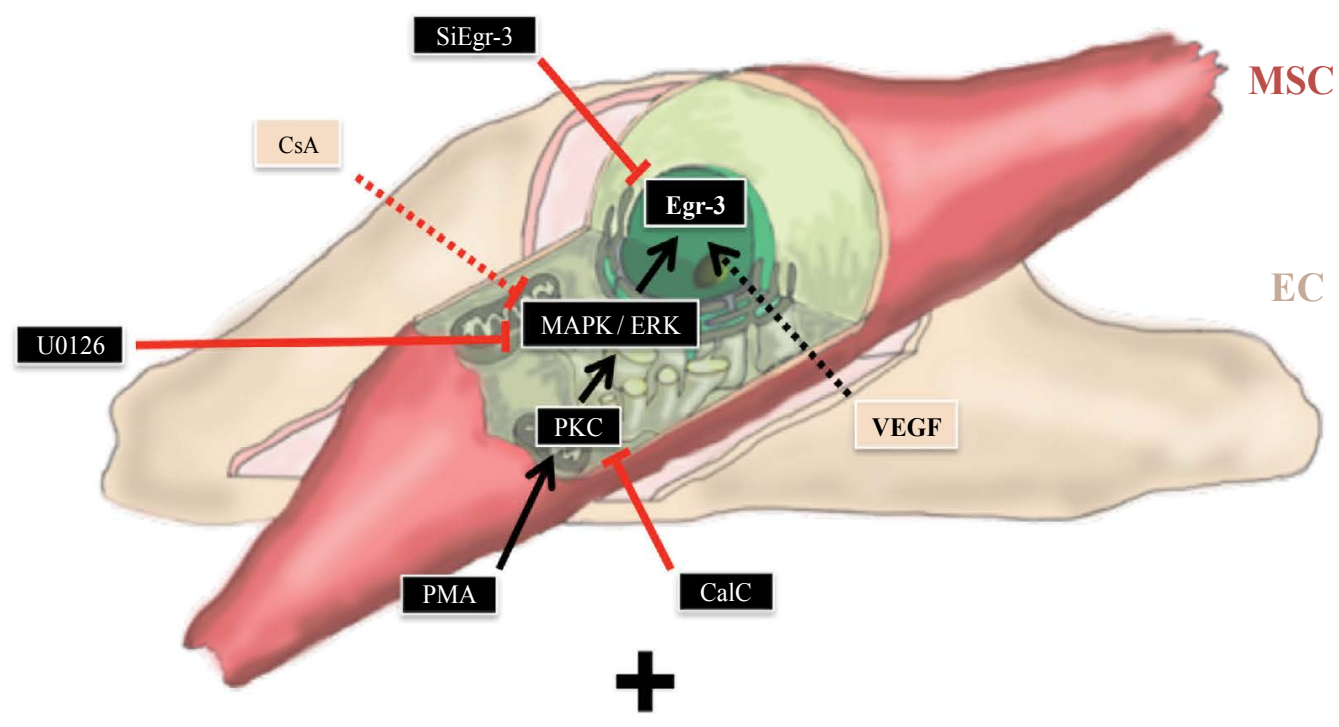

Angiogenic stimuli

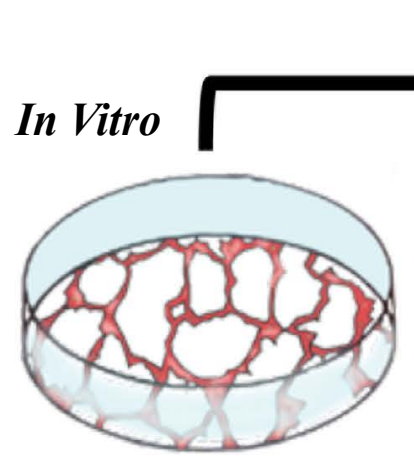

Matrigel-induced network

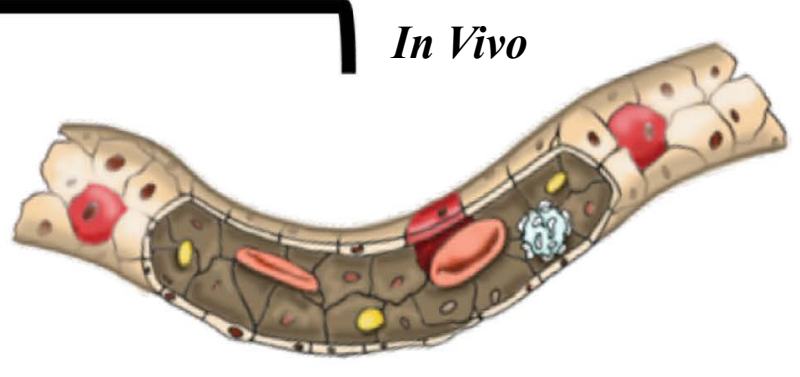

Functional microvasculature

Figure 6: Regulatory mechanisms controlling angiogenesis by multipotent MSCs and mature ECs. To regulate their overall activities, cells use a variety of clearly-defined environmental signals differentially associated with ON (black arrows) and OFF (red arrows) mechanisms, which stimulate and inhibit intracellular effectors respectively. Our results from UCBMSCs indicate that response to angiogenic stimuli is controlled by a central PKC/MAPK/ERK/Egr-3 signaling clue. In Matrigel, UCBMSCs form well-developed networks resembling last stages of in vivo angiogenesis in which they also participate actively, as we have previously demonstrated in mice where new microvasculature connected with the host circulatory system are developed after subcutaneous co-transplantation [18]. We also suggest that there are mechanisms unique to mature ECs (discontinuous lines) and common (continuous lines) to multipotent MSCs and ECs regulating angiogenesis.

lack of response by UCBMSCs may be due, in part, to the fact that these cells do not express baseline levels of VEGFR-2 [18] and do not belong to this particular cell lineage. However, Egr-3 levels declined and vascular-like network formation was inhibited following treatment with Cal C and U0126, known inhibitors of PKC $\alpha$ and MAPK/ERK (MEK) signaling $[10,15,38,39]$. ISO, which has been related to MEK activation [28], also produced a paradoxical effect; stimulating Egr-3 expression in UCBMSCs when used alone, but having an attenuating effect when used in combination with PMA. Although increase in Egr3 level following ISO treatment could be a mere artifact arising from the fact that isoproterenol and modulators of Egr-3 gene expression utilize common signaling pathways, this result could support previous observations of a regulatory mechanism based on crosstalk between $\mathrm{G}$ protein-coupled receptors and components downstream of receptor tyrosine kinases such as MAPK [40]. Thus, taken together, it would appear that independent of CsA and VEGF, Egr-3 mediated UCBMSC angiogenic behaviour through the integration of distinct signals which efficiently modulate the level of its downstream response. Further studies would be necessary to determine whether
Egr-3 expression is also modulated through both CXCR-4- and p38 mitogen-activated kinase-mediated signaling in UCBMSCs, as is the case in T lymphocytes [39]. For instance, inhibition of Egr-3-mediated activation through the p38 pathway could enhance cell differentiation towards the cardiomyogenic lineage, as suggested by previous findings using human embryonic stem cells [41].

In conclusion, we confirm that UCBMSCs constitute a valuable model for the analysis of angiogenic cell migration and assembling into growing vascular structures [18]. In a clinical context, the understanding that effective repair of the vast majority of tissues must be accompanied by the provision of an adequate vascular system has prompted an active search for cell types that could facilitate this process during therapy. Recently, together with MSCs derived from bone marrow and adipose tissue [42], UCB has been shown to be a valuable source for human regenerative medicine [17]. Particularly, UCBMSCs exhibit high capacity for migration and development of an extensive, well-organized polygonal cell network that is equivalent to that produced by HUVECs in vitro [18]. Furthermore, in mice, UCBMSCs 
Citation: Roura S, Bagó JR, Gálvez-Montón C, Blanco J, Bayes-Genis A (2013) In Vitro Characterization of the Molecular Machinery Regulating Umbilical Cord Blood Mesenchymal Stem Cell Angiogenesis: A Step Towards Multipotent Stem Cell Therapy for Vascular Regeneration. J Stem Cell Res Ther 3: 140. doi:10.4172/2157-7633.1000140

Page 9 of 10

induces the generation of microvasculature connected with the host circulatory system. When implanted with fibrin in a myocardial infarct model, UCBMSCs also organized into vascular networks, reduced scar size and increased the blood vessel-occupied area in the subjacent myocardium [18]. The findings presented here contribute to a better understanding of the molecular machinery regulating angiogenesis by multipotent MSCs [43]. Notably, we highlight that Egr-3 is commonly involved regulating mature EC and multipotent MSC angiogenesis through a central PKCa/MAPK/ERK via; this knowledge may be applied to increase efficacy of cell therapies against human diseases with vascular deficit, including cardiac alterations of ischemic origin.

\section{Acknowledgements}

We are grateful to Josep Maria Pujal and Marco A. Fernández for technical assistance in flow cytometry analysis.

\section{References}

1. Fishman AP (1982) Endothelium: a distributed organ of diverse capabilities. Ann N Y Acad Sci 401: 1-8.

2. Augustin HG, Kozian DH, Johnson RC (1994) Differentiation of endothelial cells: analysis of the constitutive and activated endothelial cell phenotypes. Bioessays 16: 901-906.

3. Edgington T (1995) Vascular biology: integrated molecular cell biology. FASEB J 9: 841-842.

4. Rodgers GM (1998) Hemostatis properties of normal and perturbed vascular cells. FASEB J 2: 116-123.

5. Carmeliet $P$ (2003) Angiogenesis in health and disease. Nat Med 9: 653-660.

6. Risau W (1997) Mechanisms of angiogenesis. Nature 386: 671-674.

7. Asahara T, Masuda H, Takahashi T, Kalka C, Pastore C, et al. (1999) Bone marrow origin of endothelial progenitor cells responsible for postnatal vasculogenesis in physiological and pathological neovascularization. Circ Res 85: 221-228.

8. Carmeliet P, Jain RK (2000) Angiogenesis in cancer and other diseases. Nature 407: 249-257.

9. Carmeliet $P$ (2005) Angiogenesis in life, disease and medicine. Nature 438: 932-936.

10. Suehiro JI, Hamakubo T, Kodama T, Aird WC, Minami T (2010) Vascular endothelial growth factor activation of endothelial cells is mediated by early growth response-3. Blood 115: 2520-2532.

11. Liu D, Jia H, Holmes DI, Stannard A, Zachary I (2003) Vascular endothelial growth factor-regulated gene expression in endothelial cells: KDR-mediated induction of Egr3 and the related nuclear receptors Nur77, Nurr1, and Nor1. Arterioscler Thromb Vasc Biol 23: 2002-2007.

12. Liu D, Evans I, Britton G, Zachary I (2008) The zinc-finger transcription factor, early growth response 3 , mediates VEGF-induced angiogenesis. Oncogene 27: 2989-2998

13. Xu H, Czerwinski P, Hortmann M, Sohn HY, Förstermann U, et al. (2008) Protein kinase $\mathrm{C}$ alpha promotes angiogenic activity of human endothelial cells via induction of vascular endothelial growth factor. Cardiovasc Res 78: 349355.

14. Roura S, Farré J, Hove-Madsen L, Prat-Vidal C, Soler-Botija C, et al. (2010) Exposure to cardiomyogenic stimuli fails to transdifferentiate human umbilical cord blood-derived mesenchymal stem cells. Basic Res Cardiol 105: 419-430.

15. Li L, Yun SH, Keblesh J, Trommer BL, Xiong H, et al. (2007) Egr3, a synaptic activity regulated transcription factor that is essential for learning and memory. Mol Cell Neurosci 35: 76-88.

16. Medhora M, Dhanasekaran A, Pratt PF Jr, Cook CR, Dunn LK, et al. (2008) Role of JNK in network formation of human lung microvascular endothelial cells. Am J Physiol Lung Cell Mol Physiol 294: L676-685.

17. Roura S, Pujal JM, Bayes-Genis A (2012) Umbilical cord blood for cardiovascular cell therapy: from promise to fact. Ann N Y Acad Sci 1254: 66-70.

18. Roura S, Bagó JR, Soler-Botija C, Pujal JM, Gálvez-Montón C, et al. (2012)
Human umbilical cord blood-derived mesenchymal stem cells promote vascular growth in vivo. PLoS One 7: e49447.

19. Prat-Vidal C, Roura S, Farré J, Gálvez C, Llach A, et al. (2007) Umbilica cord blood-derived stem cells spontaneously express cardiomyogenic traits. Transplant Proc 39: 2434-2437.

20. Livak KJ, Schmittgen TD (2001) Analysis of relative gene expression data using real-time quantitative PCR and the 2(-Delta Delta C(T)). Methods 25: 402-408.

21. Choi JH, Hur J, Yoon CH, Kim JH, Lee CS, et al. (2004) Augmentation of therapeutic angiogenesis using genetically modified human endothelial progenitor cells with altered glycogen synthase kinase-3beta activity. J Bio Chem 279: 49430-49438.

22. Rigol M, Solanes N, Farré J, Roura S, Roqué M, et al. (2010) Effects of adipose tissue-derived stem cell therapy after myocardial infarction: impact of the route of administration. J Card Fail 16: 357-366.

23. Dominici M, Le Blanc K, Mueller I, Slaper-Cortenbach I, Marini F, et al. (2006) Minimal criteria for defining multipotent mesenchymal stromal cells. The International Society for Cellular Therapy position statement. Cytotherapy 8 : 315-317.

24. Auerbach R, Lewis R, Shinners B, Kubai L, Akhtar N (2003) Angiogenesis assays: a critical overview. Clin Chem 49: 32-40.

25. Angulo J, Matou S (2007) Application of mathematical morphology to the quantification of in vitro endothelial cell organization into tubular-like structures. Cell Mol Biol (Noisy-le-grand) 53: 22-35.

26. Khoo CP, Micklem K, Watt SM (2011) A comparison of methods for quantifying angiogenesis in the Matrigel assay in vitro. Tissue Eng Part C Methods 17: 895-906.

27. Jeyaseelan K, Ma D, Armugam A (2001) Real-time detection of gene promoter activity: quantitation of toxin gene transcription. Nucleic Acids Res 29: e58.

28. Zou Y, Yao A, Zhu W, Kudoh S, Hiroi Y, et al. (2001) Isoproterenol activates extracellular signal-regulated protein kinases in cardiomyocytes through calcineurin. Circulation 104: 102-108

29. Bae YK, Sung JY, Kim YN, Kim S, Hong KM, et al. (2012) An in vivo C. elegans model system for screening EGFR-inhibiting anti-cancer drugs. PLoS One 7:e42441.

30. Abe M, Sato Y (2001) cDNA microarray analysis of the gene expression profile of VEGF-activated human umbilical vein endothelial cells. Angiogenesis 4 : 289-298.

31. Montesano R, Orci L (1985) Tumor-promoting phorbol esters induce angiogenesis in vitro. Cell 42: 469-477.

32. Morris PB, Hida T, Blackshear PJ, Klintworth GK, Swain JL (1988) Tumorpromoting phorbol esters induce angiogenesis in vivo. Am J Physiol 254: C318 322.

33. Tsopanoglou NE, Pipili-Synetos E, Maragoudakis ME (1993) Protein kinase C involvement in the regulation of angiogenesis. J Vasc Res 30: 202-208.

34. Mages HW, Stamminger T, Rilke O, Bravo R, Kroczek RA (1993) Expression of PILOT, a putative transcription factor, requires two signals and is cyclosporin $A$ sensitive in T cells. Int Immunol 5: 63-70.

35. Mittelstadt PR, Ashwell JD (1998) Cyclosporin A-sensitive transcription factor Egr-3 regulates Fas ligand expression. Mol Cell Biol 18: 3744-3751.

36. Hernández GL, Volpert OV, Iñiguez MA, Lorenzo E, Martínez-Martínez S, et al. (2001) Selective inhibition of vascular endothelial growth factor-mediated angiogenesis by cyclosporin $A$ : roles of the nuclear factor of activated $T$ cells and cyclooxygenase 2. J Exp Med 193: 607-620.

37. Rafiee P, Heidemann J, Ogawa H, Johnson NA, Fisher PJ, et al. (2004) Cyclosporin A differentially inhibits multiple steps in VEGF induced angiogenesis in human microvascular endothelial cells through altered intracellular signaling. Cell Commun Signal 2: 3.

38. Zhang M, Miller C, He Y, Martel-Pelletier J, Pelletier JP, et al. (1999) Calphostin $\mathrm{C}$ induces AP1 synthesis and AP1-dependent c-jun transactivation in normal human chondrocytes independent of protein kinase $\mathrm{C}$-alpha inhibition: possible role for c-jun N-terminal kinase. Cell Biochem 76: 290-302.

39. Tan X, Sanders P, Bolado J Jr, Whitney M (2003) Integration of G-protein coupled receptor signaling pathways for activation of a transcription factor (EGR-3). Genomics Proteomics Bioinformatics 1: 173-179. 
Citation: Roura S, Bagó JR, Gálvez-Montón C, Blanco J, Bayes-Genis A (2013) In Vitro Characterization of the Molecular Machinery Regulating Umbilical Cord Blood Mesenchymal Stem Cell Angiogenesis: A Step Towards Multipotent Stem Cell Therapy for Vascular Regeneration. J Stem Cell Res Ther 3: 140. doi:10.4172/2157-7633.1000140

Page 10 of 10

40. Natarajan K, Berk BC (2006) Crosstalk coregulation mechanisms of G proteincoupled receptors and receptor tyrosine kinases. Methods Mol Biol 332: 51-77.

41. Kempf H, Lecina M, Ting S, Zweigerdt R, Oh S (2011) Distinct regulation of mitogen-activated protein kinase activities is coupled with enhanced cardiac differentiation of human embryonic stem cells. Stem Cell Res 7: 198-209.
42. Soler-Botija C, Bagó JR, Bayes-Genis A (2012) A bird's-eye view of cell therapy and tissue engineering for cardiac regeneration. Ann N Y Acad Sci 1254: 57-65.

43. Hass R, Otte A (2012) Mesenchymal stem cells as all-round supporters in a normal and neoplastic microenvironment. Cell Commun Signal 10: 26. 